S.M. Kharit ${ }^{1}$, M.A. Okuneva ${ }^{2}$, A.A. Ruleva ${ }^{1}$, A.L. Perova ${ }^{1}$, A.S. Simakhodskii ${ }^{1}$, I.G. Chkhidzheria ${ }^{2}$, O.V. Parkov ${ }^{3}$, E.Y. Frolova ${ }^{4}$

${ }^{1}$ Research institute for pediatric infections of the Federal Biomedical Agency of Russia, Saint Petersburg

${ }^{2}$ Directorate of the Federal Service for Supervision of Consumer Rights Protection and Human Well-Being for Saint Petersburg, Russian Federation

${ }^{3}$ Healthcare committee of the Government of Saint Petersburg, Russian Federation

${ }^{4}$ Rostropovich-Vishnevskaya Foundation "For the health and future of children", Saint Petersburg, Russian Federation

\title{
Experience of implementation of the cohort pneumococcal vaccination program for infants in Saint Petersburg
}

\section{Author affiliation:}

Kharit Susanna Mikhailovna, PhD, Professor, head of the vaccinal prevention department of the Research institute for pediatric infections of the Federal Biomedical Agency of Russia (Federal State Institution)

Address: 9, Professora Popova Str., St. Petersburg, 197022; tel.: +7 (812) 346-31-42; e-mail: kharit-s@mail.ru

Article received: 19.03.2014. Accepted for publication: 14.05.2014.

The program of cohort immunization of 0-1-year-old children with a pneumococcal conjugate vaccine (PCV) has been going on in Saint Petersburg with the help of the Rostropovich-Vishnevskaya Foundation since June 2013. The need in cohort immunization against pneumococcal infection is substantiated by effectiveness of use of PCVs in previous years, when immunization therewith resulted in 9.5-fold and 1.8-fold reduction in the incidence rate of community-acquired pneumonias and acute otites mediae, respectively, at pediatric institutions. The joint program involved use of PCV13. 38,000 vaccinal doses confirm high safety thereof both for separate and combined administration. The article provides analysis of vaccination organization and demonstrates importance of individual work with the population and training of all medical professionals.

Keywords: pneumococcal infection, serotyps, prevention, vaccination, efficacy, safety, regional vaccination program, children.

Cohort immunization of infants, including premature infants, against pneumococcal infection has been taking place in Saint Petersburg since June 2013. The program is aimed at reducing morbidity, incapacitation and mortality of children caused by pneumococcal infection. The program is organized in accordance with decree No. 393 of 06.06.2013 of the Government of Saint Petersburg "On the additional immunization of children against pneumococcal infection, hepatitis A and pertussis with acellular vaccines in Saint Petersburg in 2013” and decree No. 5 of 06.06.2013 of the Chief State Medical Officer for the City of Saint Petersburg "On the vaccination of children against pneumococcal infection in Saint Petersburg". Two types of inoculations against pneumococcal infection have been used in the framework of implementing these decrees due to different sources of vaccine acquisition. Children born with low and extremely low body weight, intrauterine infections (IUIs), congenital abnormalities or HIV infection have been vaccinated from the age of 2 months; vaccines for these children have been purchased by the city districts. Term infants were vaccinated in the framework of the charity program organized in association with the Rostropovich-Vishnevskaya Foundation with the vaccines purchased by the Foundation for 35,000 term infants in Saint Petersburg. These children have been vaccinated at 7 months of age. The program has been monitored by the 
Federal Service for Surveillance on Consumer Rights Protection and Human Wellbeing of Russia and the Chief State Medical Officer of Russia. Healthcare Committee for the Government of Saint Petersburg issued directive No. 261-r on 03.07.2013 "On the organization of vaccination against pneumococcal infection in Saint Petersburg” for practical implementation of vaccination. This directive (Appendix No. 2) establishes the $2+1$ immunization pattern. The first two vaccines are administered in the first age of life at least 2 months apart; the third vaccine is administered in the second year of life, from the age of 12 months onwards, though at least 6 months after the second vaccine. If the child has been administered both PCVs before 7 months of age, the third vaccine is administered at the age of 12-15 months; if PCVs have been administered after the age of 7 months, the third vaccine is administered before 24 months of age. $2+1$ pattern was selected on the basis of recommendations of the World Health Organizations regarding the patterns used in national programs, which were published in the reference article [1]. Premature infants, children with IUIs or congenital abnormalities have undergone vaccination at the age of 2, 4 and 15 months; other children - at the age of 7, 9 and 20 months. This differentiation of patterns allowed for simpler accounting of consumption of the vaccines obtained from various sources. Taking into consideration the fact that untimely beginning of vaccination (from the age of 3 , not 2 months) results in coincidence of vaccination against pneumococcus with administration of scheduled vaccines (DTaP vaccine, vaccine against type b hemophilic infection, inactivated poliomyelitis vaccine); administration of 4 injections at almost one time creates psychological problems, what with 5 injections (when there is a coincidence with hepatitis B vaccination). This situation established a need in using combined drugs in premature infants, children with IUIs and congenital abnormalities. These children have been administered combined vaccines on the basis of the acellular pertussis vaccine at the expense of the Saint Petersburg additional immunization program. A 13-valent vaccine against pneumococcal infection has been administered in the city. Results of microbiological studies have served as the basis therefor. Thus, S.V. Sidorenko et al. [2] performed a prospective non-interventional hospital epidemiological study in Saint Petersburg to analyze serotypical composition of the Streptococcus pneumoniae inducing meningites, community-acquired pneumonia and acute otitis media and coverage of the circulating serotypes with pneumococcal conjugate vaccines (PCVs) varying in composition (PCV7, 10, 13). The main S. pneumoniae serotypes inducing purulent meningitis in children are serotypes 19F, 14 and serogroup 6. Coverage of the purulent meningitis-inducing S. pneumoniae serotypes with PCV7 is 70.6\%, with PCV10 and PCV13 - 76.5\%. The main acute otitis media-inducing pneumococcal serotypes in Saint Petersburg are serotypes 19F, 3, 23F and serogroup 6. Coverage with PCV7 and PCV10 is the same $-63.2 \%$ of 0 -2-year-old children and $32.5 \%$ of 5-17-year-old children. These parameters for PCV12 are 79 and 55\%, respectively. PCV7 and PCV10 produced the same coverage in the event of community-acquired pneumonia: $57.1 \%$ in children and 56.1\% in adults. PCV13 featured better parameters: by $14.3 \%$ in children and by $34.5 \%$ in adults. The obtained data substantiated reasonability of using pneumococcal conjugate vaccines for cohort immunization in Saint Petersburg; PCV13 provided the best coverage of the $S$. pneumoniae serotypes inducing the main pneumococcal diseases. It ought to be mentioned that similar serotype distribution was observed in other regions of Russia as well $[3,4]$. The need in cohort use of PCVs for vaccinating infants was substantiated by results of a preliminary monitoring of effectiveness of the PCV7, which was used in previous years in the framework of the program "City for premature infants". Comparative evaluation of immunization results in respect of the incidence rate of otites and pneumonias at one of the infant orphanages (and non-vaccinated children) demonstrated high effectiveness of vaccination for pneumonia prevention. 50 children were administered the 7-valent pneumococcal conjugate vaccine. According to the anamnesis, 24\% (12) of the children were healthy, whereas $76.0 \%$ (38) had various diseases, including allergic diseases (16\% [8]); neurological diseases, such as residual-organic damage of the central nervous system, hydrocephalus, cerebral palsy, symptomatic epilepsy and perinatal encephalopathy (10\% [5]); ENT pathologies, such as chronic adenoiditis and chronic tonsillitis (4\% [2]); somatic diseases - hypotrophy (4\% [2]) and 
bronchopulmonary dysplasia (2\% [1]); and concomitant pathologies, such as IUIs, CNS damage, allergic manifestations, frequent diseases (40\% [20]). Preventive effectiveness of PCV7 was determined on the basis of the difference between incidence rates of acute otitis media and community-acquired pneumonia in the group of vaccinated patients $(n=50)$ and the control group $(n=100)$. No differences in age, concurrent pathologies and risk factors of pneumococcal infection development were observed. All the children remained in the same social and living conditions of one closed organized group of children for 1-4 years of observation. The calculated incidence rate of pneumonias of any etiology per 1,000 children in the group of vaccinated patients was lower throughout the observation period (pic. 1). Taking into consideration person-years, pneumonia incidence rate in the group of vaccinated patients was 9.7 per 1,000 person-years (95\% CI - 9.1-10.3) throughout the observation period, in the control group 92.6 per 1,000 person-years (95\% CI - 91.3-93.9). Effectiveness index of vaccination against pneumonias of any etiology was 9.5 , effectiveness coefficient $-89.5 \%$.

Analysis of the calculated incidence rate of otites revealed downward trend by the $4^{\text {th }}$ year of observation in both groups, probably, due to children's growing up; still, this rate was always higher in the non-vaccinated patients. Incidence rate was 1.8 lower in the main group than in the control group - 155.3 (95\% CI - 150.9-155.7) and 263.9 (95\% CI - 261.7-266.1) per 1,000 person-years. Effectiveness index and coefficient of vaccination against acute otitis media were 1.8 and $44.3 \%$. Thus, the vaccination against pneumonias proved to be more effective than the vaccination against acute otitis media; this agrees with the data quoted in the reference article of the World Health Organization [1].

Various organizational measures have been performed in order to implement the program of cohort immunization against pneumococcus in the city. Meetings and seminars with heads of public health authorities and the Federal Service for Surveillance on Consumer Rights Protection and Human Wellbeing of Russia and public health workers from pediatric polyclinics have been held. Account of vaccines and reports has been arranged on the lowest (polyclinics) and the highest (Healthcare Committee, Directorate of the Federal Service for Supervision of Consumer Rights Protection and Human Well-Being of Russia and the Rostropovich-Vishnevskaya Foundation) levels. Sessions of district emergency sanitary and anti-epidemic commissions and the Council of heads of administrations have been held with heads of district administrations; informational letters have been issued. Meetings with heads of public health authorities and head doctors of healthcare establishments have been held; the data have been quoted in informational letters and bulletins. Continuous awareness-building work with the population has been conducted, involving use of web-sites of district administrations, printed sources of municipal units, information of medical and preventive facilities, classes at the Young Mothers' School, conversations during social welfare events and attendances and the "Health Corner" booklets and stands.

Educational seminars on the issues of pneumococcal infection and description of the vaccine's safety and reactogenicity have been held at all the pediatric polyclinics for public health workers. The vaccinated children would have been observed for the first 1-3 days, similarly to the patients vaccinated with inactivated vaccines. All the cases of severe reactions with fever over $38.6{ }^{\circ} \mathrm{C}$ have been routinely registered in registers of medical and preventive facilities and, if a post-vaccinal complication had been suspected, in the city epidemic bureau's register. 1 complaint of a post-vaccinal complication has yet been filed to the Federal Service for Supervision of Consumer Rights Protection and Human Well-Being of Russia; however, investigation of that episode helped to diagnose an acute (intercurrent) disease, which was not associated with vaccination. Normal vaccinal process course did not differ considerably from the previously known data on PCV7 administration [5].

The project started in June 2013. It has been planned to vaccinate 42,500 term infants born in 2013 in 19 districts of Saint Petersburg. 23,330 term infants, who have attained the age of 7 months by July 2013 (born in December 2012) were registered in the period from July 2013 to January 2014. By 31.05.2014, 20,382 of them had been vaccinated with the first dose, 
$14,178(69.5 \%)$ - with the second dose. By the same date, 2,443 premature infants with IUIs or congenital abnormalities had been vaccinated with the $1^{\text {st }}$ dose; 1,806 of them were vaccinated with the second date (73.9\% [81.4\% of the required amount], pic. 2). Higher vaccination rate of the children with risk factors may have been caused by their vaccination with other Immunization Calendar vaccines at the scheduled immunization time, which is why they have been administered combined vaccines on the basis of the acellular pertussis vaccine at the same time.

The experience accumulated on cohort introduction of PCVs for children of 0-2 years of age indicates that the successful implementation requires preliminary and systemic informational work with parents, methodological work with public health works and control over the timely immunization of children at polyclinics.

\section{REFERENCES}

1. Pneumococcal vaccines. Who position paper - 2012. Weekly epidemiological record. 6 april 2012; 14 (87): 129-144. Available at: http://www.who.int/wer

2. Lobzin Yu. V., Sidorenko S. V., Kharit S. M., Belanov S. S., Volkova M. O., Gostev V. V., Alekseenko S. I., Petrova I., Sergeeva E. V., Koroleva I. S., Orlov A. V., Frolova E.Ya. Infektologiya - Infectology. 2013; 5 (4): 36-42.

3. Tatochenko V., Sidorenko S., Namazova-Baranova L., Mayan s kiy N., Kulichenko T., Baranov A., Lobzin Y., Kharit S., Kozlov R., Andreeva I., Muravjev A., Chagaryan A., Koroleva I., Beloshitskiy G., Mironov K., Degtyareva E., Reinert R. R. Streptococcus pneumoniae serotype distribution in children in the Russian Federation before the introduction of pneumococcal conjugate vaccines into the National Immunization Program. Expert Rev Vaccines. 2014; 13 (2): 257-264.

4. Mayanskii N. A., Alyab'eva N. M., Ivanenko A. M., Ponomarenko O. A., Katosova L. K., Lazareva A. V., Kulichenko T. V., Namazova-Baranova L. S. Voprosy diagnostiki v pediatrii Diagnostics in pediatrics. 2013; 5 (3): 5-13.

5. Ruleva A. A., Kharit S. M., Snegova N. F., Vavilova V. P., Vaiman O. A., Mamet'eva V. L., Andreeva E. A., Vavilova T. A. Voprosy sovremennoi pediatrii - Current pediatrics. 2010; 9 (2): 120-124.

Pic. 1. Dynamics of incidences rates of pneumonias (1) and otites (2) in the vaccinated patients and the control group
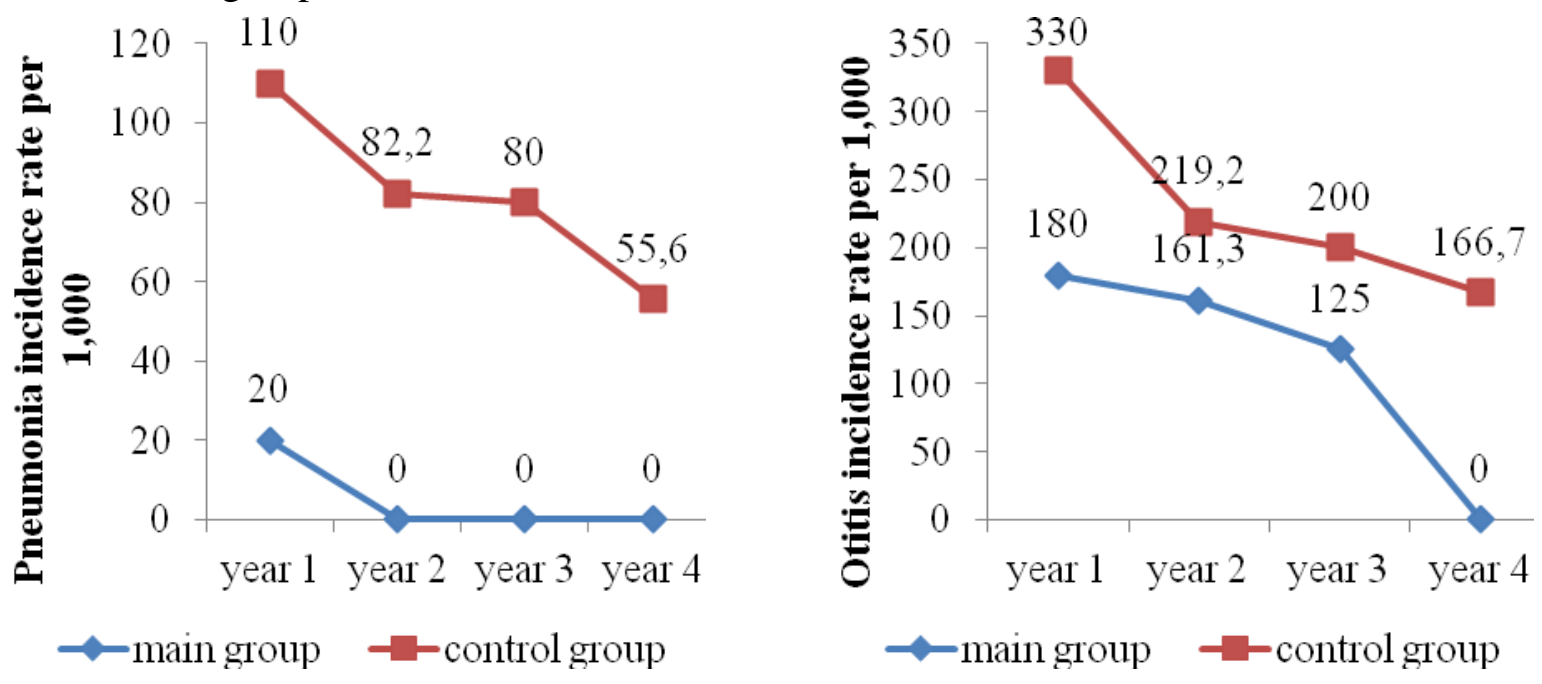

Pic. 2. PCV3 immunization rate of children in Saint Petersburg in 2014 
Prevenar 13 account and immunization rate in premature infants and risk groups by 30.04.2014 in Saint Petersburg districts

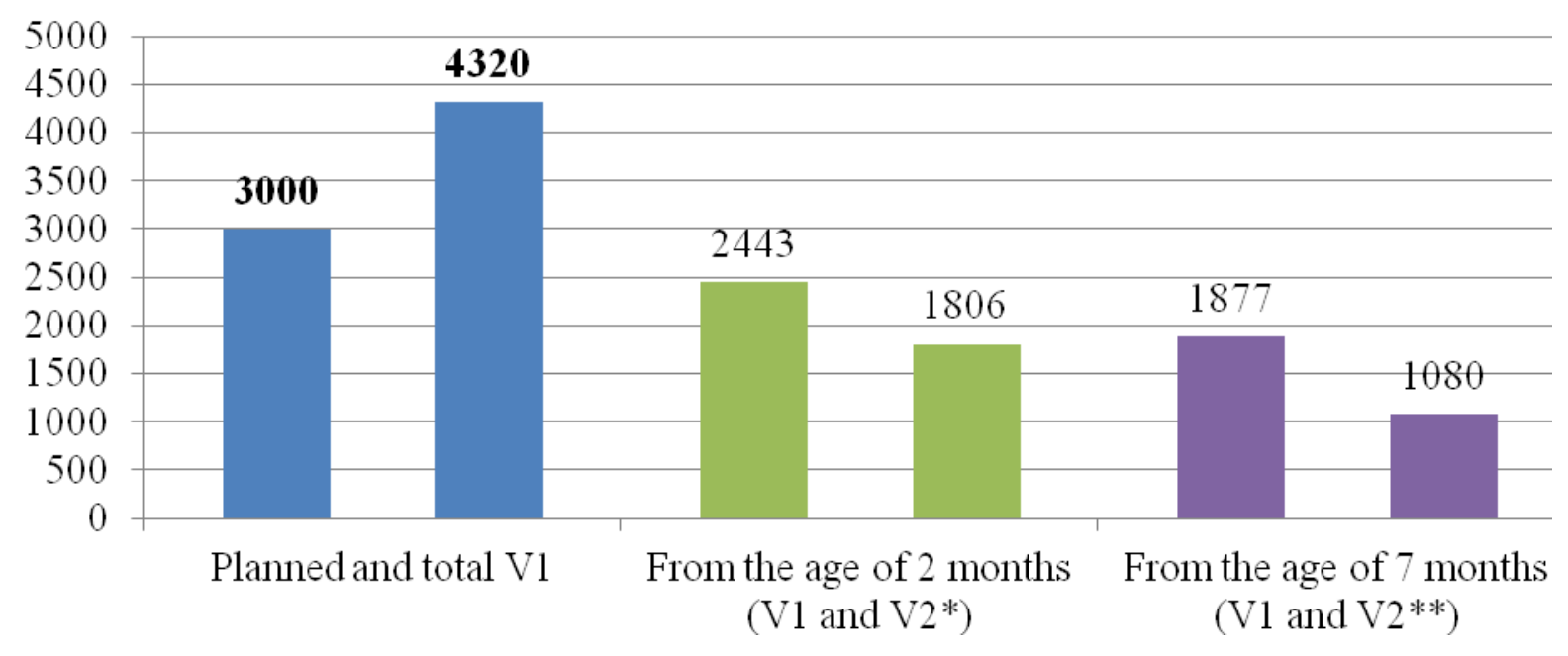

Note. * $-81.4 \%$ of the required amount, ** $-63.7 \%$ of the required amount. 